\title{
Three-body forces in Bethe-Salpeter and light-front equations
}

\author{
V.A. Karmanov* \\ Lebedev Physical Institute, Moscow, Russia \\ E-mail: karmanov@lpsc.in2p3.fr

\section{P. Maris}

Iowa State University, Ames, Iowa, USA

\begin{abstract}
In relativistic frameworks, given by the Bethe-Salpeter and light-front bound state equations, the binding energies of system of three scalar particles interacting by scalar exchange particles are calculated. In contrast to two-body systems, the three-body binding energies obtained in these two approaches differ significantly from each other: the ladder kernel in light-front dynamics underbinds by approximately a factor of two compared to the ladder Bethe-Salpeter equation. By taking into account three-body forces in the light-front approach, generated by two exchange particles in flight, we find that most of this difference disappears; for small exchange masses, the obtained binding energies coincide with each other.
\end{abstract}

LIGHT CONE 2008 Relativistic Nuclear and Particle Physics

July 7-11, 2008

Mulhouse, France

${ }^{*}$ Speaker. 


\section{Introduction}

In this article we sketch out the results of our theoretical study [1] of three-body bound states, carried out in full relativistic framework, given by the Bethe-Salpeter (BS) approach [2] and lightfront dynamics (LFD) [3, 4]. Eventually, the goal is to investigate the bound states of fermions, using e.g. a meson-exchange model in the case of 3-nucleon bound states (triton, ${ }^{3} \mathrm{He}$ ), and baryons as bound states of three (non-perturbatively dressed) quarks interacting via gluons, and calculate their electromagnetic form factors. For simplicity however, and as a first step, we restrict ourselves to spinless system. For such system, the three-body BS equation with one-boson exchange interaction was first solved and the results were presented in [5]. As far as we know, the LFD three-body equation with one-boson exchange kernel has not yet been solved. Previously, the three-body LFD equation was solved in $[6,7]$ for zero-range interaction. Its solution was also found in the relativistic quantum-mechanical approach for bound and scattering states [8], with a phenomenological mass operator.

Though a system of spinless particles is oversimplified, some its properties are rather general and their study gives useful physical insight. Especially, the comparison of results found in the BS and LFD approaches is fruitful. An important observation is the fact that for two-body system, these two approaches give results very close to each other both for the binding energy [9] and also for electromagnetic form factors [10]. The comparison of BS and LFD calculations, for the constituent mass $m=1$ and for the exchange mass also $\mu=1$, is shown in fig. 1 . The curve NR means nonrelativistic binding energy (obtained via Schrödinger equation), which considerably differs, even for weakly bound system, from almost coinciding with each other BS and LFS binding energies.

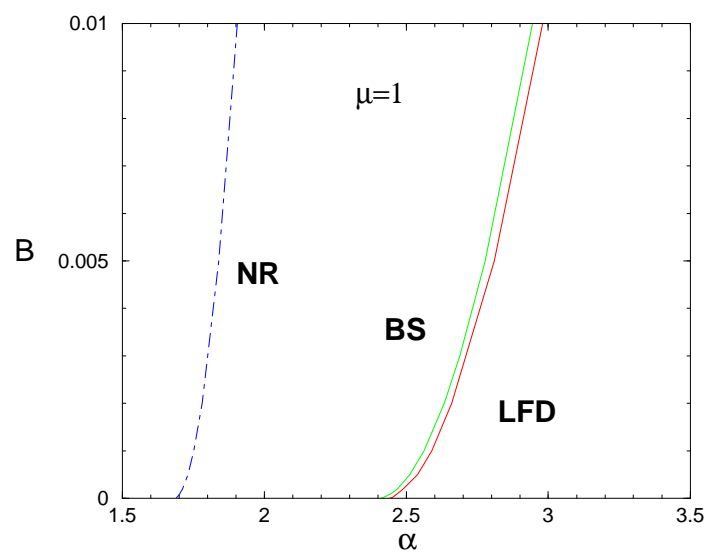

Figure 1: Binding energy $B$ of system of two spinless particles interacting by one-boson exchange with mass $\mu=1$ v.s. the coupling constant $\alpha=g^{2} /\left(16 \pi \mathrm{m}^{2}\right)$, calculated by BS, LFD and Schrödinger equations. The units are set by $m=1$. The figure is taken from [9].

It is important to note, however, that the actual ladder kernels in the BS approach and in LFD are not identical, nor are they given by the same graphs. In the BS ladder kernel, there is no notion of time-ordering in the diagrams. On the other hand, the kernel for the LFD equation is given by the time-ordered graphs in the light-front (LF) time. Thus, the second iteration in the two-body ladder BS equation (the second-order Feynman box graph, figure 2(a)), when represented as a set of timeordered graphs, turns into six LF time-ordered graphs, including two so-called "stretched boxes" 


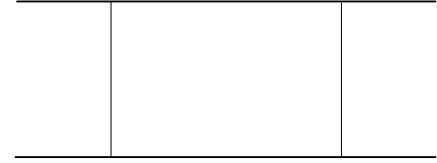

(a)

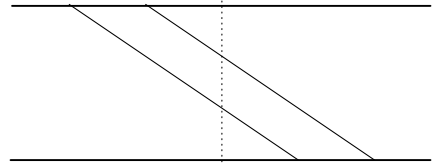

(b)

Figure 2: (a) Feynman ladder graph with two exchanges. (b) One of six time-ordered graphs ("stretched box"), generated by the ladder Feynman graph (a).

with two exchange particles in the intermediate state. One of such stretched boxes is shown in figure 2(b). These stretched boxes with two (and more) exchange particles in the intermediate state are implicitly included in the BS equation, but they are not generated by iterations of the LF ladder kernel; therefore, they are omitted in the corresponding LFD bound state equation. In principle, this will cause a difference between the BS and LFD results. However, direct examination of the stretched boxes $[11,12]$ shows that they are small. This explains, why the BS and LFD two-body binding energies are very close to each other, despite the fact that the kernels are not identical.

We will see below that in the three-body problem the situation is quite different: even with a simple one-boson exchange kernel, the binding energies obtained by BS and LFD significantly differ from each other, in drastic contrast to two-body case. This difference will be explained by the three-body forces generated by two exchange particles in fight.

\section{Three-body results for ladder kernel}

We consider a scalar field theory, with interaction $-g \phi^{2} \varphi$, and the bound states consisting of three particles $\phi$, interacting via the exchange of a $\varphi$ boson. Three-body equation is graphically shown in fig. 3.

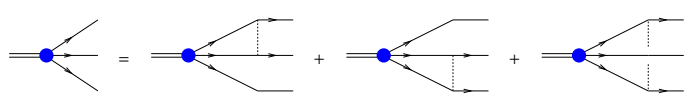

Figure 3: Three-body bound state equation with ladder kernel.

The one-boson exchange BS kernel is simply

$$
K\left(k, k^{\prime}\right)=\frac{-g^{2}}{\left(k-k^{\prime}\right)^{2}-\mu^{2}+i \varepsilon} .
$$

In LFD, this kernel is represented through LF variables $\vec{k}_{\perp}, x$ (see e.g. eq. (7.27) from [3]). Exactly the same kernels were used in the two-body BS and LFD equations. On energy shell, the expressions for BS and LFD kernels, being represented in terms of the same variables, are identical. Whereas off energy shell they differ from each other.

The three-body BS amplitude $\Gamma(p, q ; P)$ ( $p$ and $q$ are the Jacobi four-momenta) depends on five scalar variables: $p^{2}, q^{2}, p \cdot q, p \cdot P, q \cdot P$. The square $P^{2}=M^{2}$ is fixed by the value of the bound mass $M$. To solve the equation numerically, we transform it to Euclidean space. 


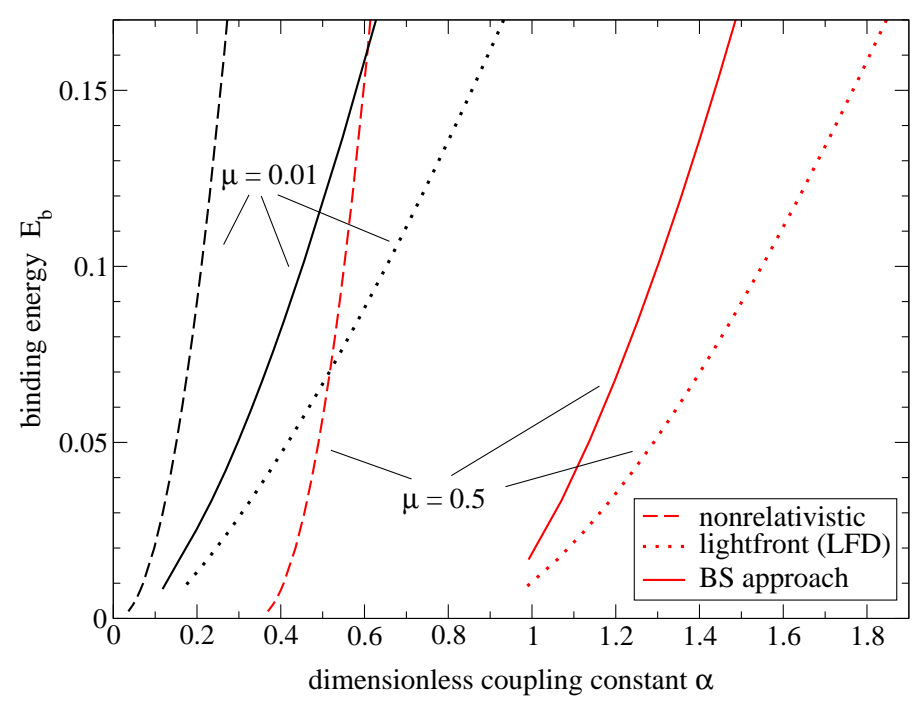

Figure 4: Three-body binding energy for one boson exchange with masses $\mu=0.01$ and $\mu=0.5$ (units set by $m=1$ ). Solid curves are the BS result; dotted curves are the LFD results; dashed curves are the solution of the non-relativistic Schrödinger equation.

To parametrize the three-body LF wave function $\psi\left(\vec{k}_{12 \perp}, x_{12} ; \vec{k}_{3 \perp}, x_{3}\right)$, we introduce transverse momenta $\vec{k}_{\perp}$ and the ratios $x$ and construct from them LF Jacobi variables: $\vec{k}_{12 \perp}, x_{12}$ are defined for two-body subsystem $12, \vec{k}_{3 \perp}, x_{3}$ are momenta of the 3 rd particle. The LF wave function also depends of five scalar variables: $\vec{k}_{12 \perp}^{2}, \vec{k}_{3 \perp}^{2}, \vec{k}_{12 \perp} \cdot \vec{k}_{3 \perp}, x_{12}, x_{3}$.

Thus we have an integral equation, which is four-dimensional in the BS approach and threedimensional in LFD, for the bound state amplitude which is a function of five independent variables in both cases. We solve it numerically by straightforward discretization of the internal and external variables; we do not make use of any expansion of $\Gamma$ and $\psi$ in partial waves, nor any other set of basis functions.

For small binding energies $E_{b}=3 m-M_{3}$, and for two exchange masses $\mu=0.01$ and $\mu=0.5$, our results are shown in figure 4 (the units are set by $m=1$ ). For comparison, we also include the non-relativistic result obtained by solving the non-relativistic Faddeev equation in the momentum space. Clearly, all three calculations give different results. We emphasize the large difference between BS and LFD three-body binding energies, in contrast to coincidence of the two-body ones, shown in fig. 1.

In this way, new interesting and important features emerge, highlighting differences between the BS approach and LFD. In a three-body problem the difference between LFD and time-ordered iterated BS kernels cannot be reduced to (negligible) stretched boxes: new LFD (non-loop) diagrams appear, which are shown in figure 5. These graphs correspond to three-body forces which are not taken into account in the three-body LFD equation with ladder kernel. On the other hand, they are implicitly included in the three-body BS equation.

The three-body potential, resulted from the first-order relativistic corrections due to the first eight graphs of figure 5, was first found in Ref. [13]. Its contribution to the triton binding energy was calculated in Ref. [14]. However, in Ref. [15] it was shown that this relativistic correction cancels with the corresponding relativistic correction to the second iteration of the one-boson exchange. 

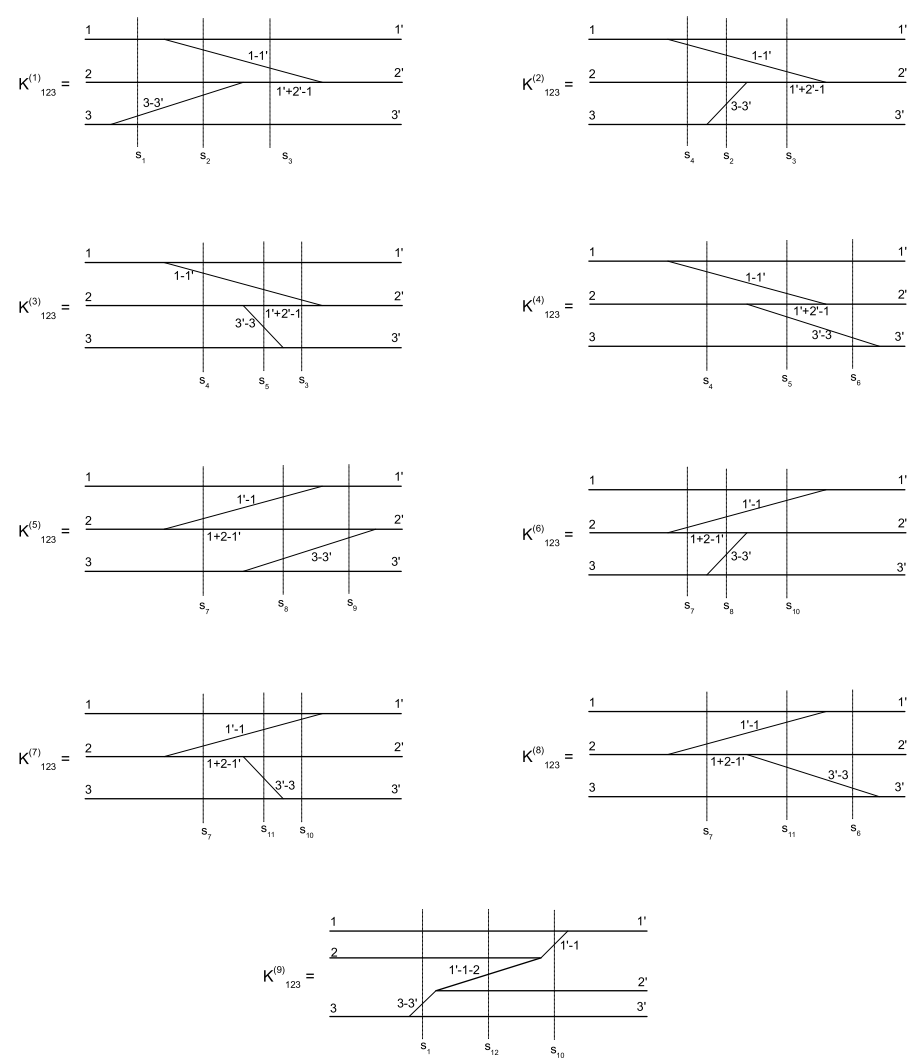

Figure 5: Contribution to three-body forces from two bosons in flight $\left(K_{123}^{(1-8)}\right)$ and from pair-creation $\left(K_{123}^{(9)}\right)$.

Due to this cancellation, sum of these two corrections does not contribute in the Schrödinger equation. However, in truly relativistic framework, the full graphs figure 5 (not a first-order relativistic correction) should be taken into account.

The next question is: Can the difference between three-body BS and LFD results be explained by the diagrams of figure 5? In order to answer this question, we performed a perturbative calculation of the correction to the LFD bound state mass $M_{3, L F}^{2}$ due to these three-body forces. That is, we calculated the set of LFD graphs shown in fig. 5 and then found perturbatively the corresponding correction to the LFD binding energy.

The three-body bound state mass squared $M_{3}^{2}$ for the exchange masses $\mu=0.01,0.5$ and 1.5 are shown in figure 6. Like in figure 4, the solid and dotted curves are our results from the three-body BS and LF bound state equations with the two-body one-boson exchange kernel. As in figure 4 for binding energy, the BS and LFD masses squared differ significantly from each other and the difference increases with increase of the binding energy (and, hence, with the coupling constant).

The diamonds with error bars in figure 6 indicate the sum $M_{3, L F}^{2}+\Delta M_{3}^{2}$, where $\Delta M_{3}^{2}$ is the correction due to the diagrams of figure 5 , calculated perturbatively. The errors indicate the numerical uncertainty, mainly due to the numerical evaluation of a 12-dimensional integral $\Delta M_{3}^{2} \sim$ $\int \psi K_{123} \psi \ldots$. This correction does indeed shift the LFD three-body mass squared so that it comes in reasonably good agreement with the BS results. This is the effect of relativistic three-body forces, 

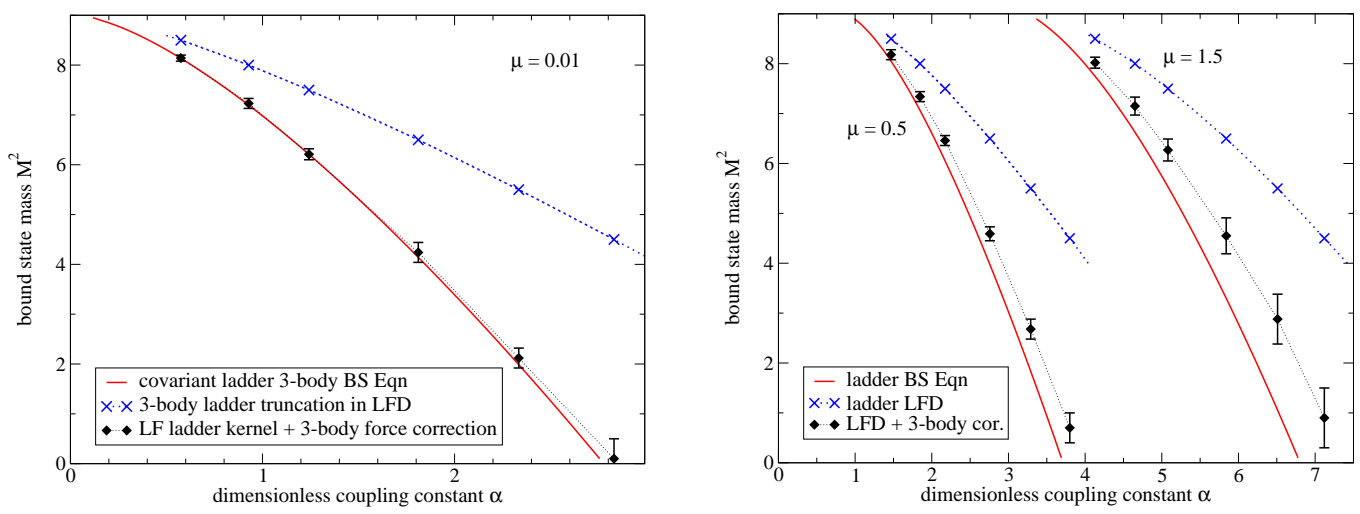

Figure 6: Three-body bound state mass squared $M_{3}^{2}$ vs. coupling constant $\alpha=g^{2} /\left(16 \pi m^{2}\right)$ for exchange masses $\mu=0.01$ (top), 0.5 and 1.5 (bottom). The units are set by the constituent mass: $m=1$.

dominated here by two exchange-bosons in flight, with a small contribution from the creation (and annihilation) of a pair of constituent particle-antiparticles.

One should of course keep in mind that here we only present a perturbative estimate of the correction due to these effective 3-body forces in LFD. Note that the perturbative calculation is unexpectedly good even for large correction compared to the value of $M^{2}$ itself, especially for small values $\mu$ of the mass of the exchange particle. The agreement becomes less pronounced as the exchange mass increases, once the exchange mass becomes larger than the constituent mass. E.g. if the exchange mass is three times the constituent mass, the perturbative correction explains about $70 \%$ of the difference in $M_{3}^{2}$ between the BS approach and LFD.

Since the corrections are substantial, one could raise the question: How reliable is a perturbative calculation in this context? To answer this question, one can solve three-body LFD equation beyond the perturbative framework, which requires significantly more work. Nevertheless, we have ample evidence that the contributions of effective 3-body forces in LFD are substantial in the threebody systems, and can explain most of the difference between the results found in the BS approach and in LFD.

\section{Conclusion}

We have solved, for the first time, the three-body BS and LFD bound state equations with a one-boson exchange kernel and found a significant difference in the corresponding binding energies. This difference is absent in the two-body BS and LFD equations [9]. After incorporating, in the LFD framework, the three-body forces of relativistic origin, generated by two exchange particles in flight, the BS and LFD three-body binding energies become very close to each other.

In the case of three-nucleon system, there are many other sources of three-body forces. Part of them is generated by the transitions $N \leftrightarrow \Delta$. In addition, there can be intrinsic three-body forces, such as the six-nucleon contact term $N N N \rightarrow N N N$, generated by chiral perturbation theory for the effective nucleon interaction, or, in QCD, three-body forces due to the triple gluon vertex. In any case, the two exchange particles in flight are present and give important contributions. The influence of such three-body forces on observables significantly exceeds the experimental precision. 


\section{Acknowledgments}

The authors are sincerely grateful to J. Vary for his interest to this work, fruitful discussions and support. One of the authors (V.A.K.) is indebted for the warm hospitality of the nuclear physics group of the Iowa State University (Ames, USA), where part of the present work was performed. This work was supported in part by the U.S. Department of Energy Grant DE-FG02-87ER40371.

\section{References}

[1] V.A. Karmanov and P. Maris, Manifestation of three-body forces in three-body Bethe-Salpeter and light-front equations, to be published.

[2] E.E. Salpeter, H.A. Bethe, A Relativistic Equation for Bound-State Problems, Phys. Rev. 84 (1951) 1232.

[3] J. Carbonell, B. Desplanques, V.A. Karmanov and J.-F. Mathiot, Explicitly Covariant Light-Front Dynamics and Relativistic Few-Body Systems, Phys. Reports 300 (1998) 215 [nucl-th/ 9804029 ].

[4] S. Brodsky, H.-C. Pauli and S. Pinsky, Quantum Chromodynamics and Other Field Theories on the Light Cone, Phys. Reports 301 (1998) 299 [hep-ph/ 9705477$].$

[5] P. Maris, Two and three-body bound states in an explicitly covariant framework, presented at the international workshorp LC2006: Light Cone QCD and nonperturbative hadron physics, Minneapolis, USA, May 15-19, 2006.

[6] T. Frederico, Null-plane model of three bosons with zero-range interaction, Phys. Lett. B 282 (1992) 409.

[7] J. Carbonell and V.A. Karmanov, Three-boson relativistic bound states with zero-range interaction, Phys. Rev. C 67 (2003) 037001 [nucl-th/ 0207073 ].

[8] T. Lin, Ch. Elster, W.N. Polyzou and W. Glöckle, First order relativistic three-body scattering, Phys. Rev. C 76 (2007) 014010 [nucl-th/ 0702005 ];

H. Witala, J. Golak, R. Skibinski, W. Glöckle, W.N. Polyzou and H. Kamada, Relativity and the low energy nd Ay puzzle, Phys. Rev. C 77 (2008) 034004 [arXiv:0801.0367 (nucl-th) ].

[9] M. Mangin-Brinet and J. Carbonell, Solutions of the Wick-Cutkosky model in the light front dynamics, Phys. Lett. B 474 (2000) 237 [nucl-th/9912050].

[10] J. Carbonell, V.A. Karmanov and M. Mangin-Brinet, Electromagnetic form factor via Bethe-Salpeter amplitude in Minkowski space, [arXiv:0809.3678 (hep-ph) ].

[11] N.C.J. Schoonderwoerd, B.L.G. Bakker and V.A. Karmanov, Entanglement of Fock-space expansion and covariance in light-front Hamiltonian dynamics, Phys. Rev. C 58 (1998) 3093

[nucl-th/9806365].

[12] J. Carbonell and V.A. Karmanov, Cross-ladder effects in Bethe-Salpeter and Light-Front equations, Eur. Phys. J. A 27 (2006) 11 [hep-th/ 0505262$].$

[13] K.A. Brueckner, C.A. Levinson, and H.M. Mahmoud, Two-Body Forces and Nuclear Saturation. I. Central Forces, Phys. Rev. 95 (1954) 217.

[14] Shin-Nan Yang, Three-body force in the triton, Phys. Rev. C 10 (1974) 2067.

[15] Shin-Nan Yang and W. Glöckle, Three-body mesonic retardation effect, Phys. Rev. C 33 (1986) 1774. 